\title{
Fuzzy Control of Fin Stabilizer at Zero Speed Based on Improved Genetic Algorithm
}

\author{
Hongzhang Jin Xiaofei Zhang Dongsong Li Zhigang Qi \\ College of Automation, Harbin Engineering University, Harbin 150001, P. R. China
}

\begin{abstract}
Frequency spectrum of ocean wave is analyzed to enhance the efficiency of fin stabilizer when the intensity of wave is changing. Random optimal control system with quadratic performance index is designed based on the extended state functions. Performance index weight matrices are optimized with genetic algorithm to find the best matching point between energy consumption and effect of roll reduction. Basic genetic algorithm is improved by analyzing characteristic of the system. Random optimal control in different sea levels is realized by introducing fuzzy control rules for weight matrices. Simulation results indicate that roll movement of ship can be reduced with least energy if weight matrices are fixed according to external disturbances.
\end{abstract}

Keywords: Genetic algorithm, Fuzzy control, Random optimization algorithm, Fin stabilizer at zero speed

\section{Instruction}

Nowadays more and more ship motion control systems are applied in various ships. Among ship motions, roll motion causes serious damages to the equipments of ships and their performance, and passengers feel uncomfortable when ships roll periodically with waves. Many efforts have been made to invent or create some equipment to weaken these bad effects. U-type tanks and fin stabilizers are most practical ones among these

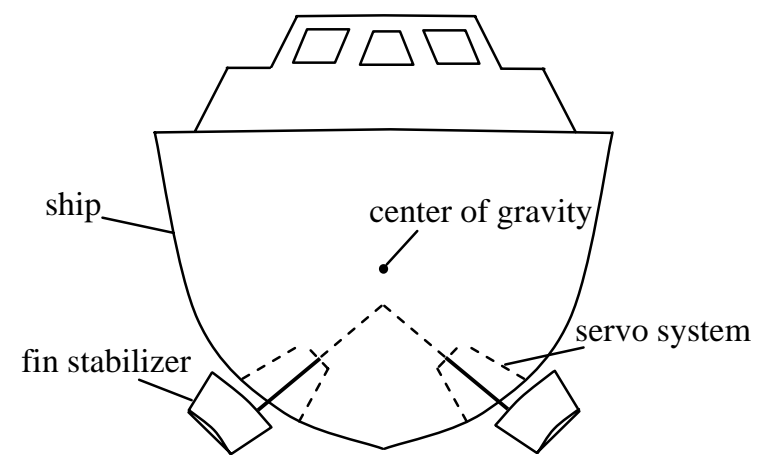

Fig. 1: Front view of a ship with fin stabilizers. equipments. But the volume of anti-roll tanks is usually too large for most ships. The owners of ships have to reduce the space for cargos to install them. Fin stabilizers are much smaller than tanks, so they have been the most widely used equipments for roll reduction. Fin stabilizers are installed on both sides of ships, the position of fin stabilizers are shown in Fig.1.

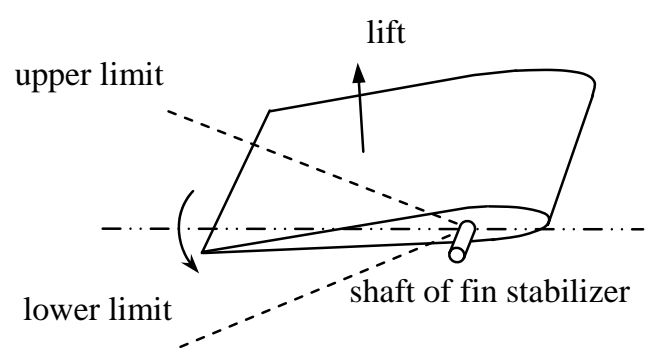

Fig. 2: Principle of fin stabilizer at zero speed.

The task of modern special type ships is more and more complex. To work on a certain point of sea level, ships must have excellent stability when anchored. So the problem of roll reduction at zero speed must be studied deeply. Anti-roll tanks can work well when ships are anchored, but the volume of anti-roll tanks is usually too large. Though Fin stabilizers are smaller than tanks, they cannot work when the ship is anchored. No lift can be produced to reduce roll motion when the ship is anchored, because the principle of fin stabilizers is similar to airfoils of plane. In order to solve this problem, some research institutes in the US and Netherlands begin to explore in this field in recent years [1]-[3]. The roll reduction and ships control institute of Harbin Engineering University also has been researching on the subject since 2005. Nowadays, the most feasible scheme is to make fin stabilizers work in traditional way when the ship sails at high speed and reduce roll motion by vibrating fin stabilizers periodically when the ship is anchored. The principle of fin stabilizer at zero speed is shown in Fig.2. Thus roll motion can be reduced in any conditions with only one set of fin stabilizers. Quantum Marine Engineering Corporation in the US has developed a series of fin stabilizers based on the 
scheme mentioned above [1]. These stabilizers are mostly equipped on yachts for the limit of driving energy. The yachts anchored in a harbor will roll with waves. Fin stabilizers at zero speed are proved to be able to reduce the roll motion remarkably and passengers feel more comfortable than ever.

For traditional fin stabilizers, the flow field around them is steady. The lift can be calculated in the same way with airfoils. For fin stabilizers at zero speed, the flow field is unsteady. The lift cannot be calculated in potential flow theory, so the classification and principle of forces on the fins must be analyzed in detail. Traditional fins are in steady flow and the forces can be decomposed into resistance along flow and lift that is vertical to the flow. The forces on fin stabilizers at zero speed in unsteady flow are similar with the tail fin of bionic fish. The difference between them is that resistance produced by rotating is concerned for fin stabilizers; however, the thrust in the direction of going forward is concerned for bionic fishes. The forces on tail fin of bionic fish are analyzed by many scholars. It is usually recognized that the forces can be divided into three types, namely shape resistance, added mass force and vortex force. The model of lift will be established by analyzing the forces in hydrodynamics.

Some new problems are brought forward during the process of application because of the special operating modes of fin stabilizers at zero speed. What is mostly concerned is driving energy. The fins move with the ship when they work in traditional way. Servo system just rotates the fins to certain positions. Energy for roll reduction mainly comes from the engine of the ship. When the ship is anchored, energy mainly comes from electro-hydraulic servo system. But the power of most power plants on present ships is limited. That is to say, the power of servo system is much lower than that of engine. The result is that the effectiveness of roll reduction is not very well when the ocean wave is intense. We can increase the capacity of the power plants on ships to solve this problem, but this method not only enhances the energy consumption but also causes the abrasion of mechanical system. So the driving energy must be limited as possible as we can. In order to solve this problem, optimal controller must be designed based on the frequency spectrum of random waves to optimize the energy consumption of servo system. The intensity of waves and the parameters of ship vary with time, so the effectiveness of fin stabilizer should be evaluated by index changing with external environment. Thus the index could be the least in any conditions. For this purpose, adaptive rules for index are presented. The weight matrices of index is optimized based on improved genetic algorithm. Then weight matrices for different intensity of ocean waves are gained.

\section{Mathematical model of roll movement}

Nowadays the widely used rolling model of ship in engineering is Conolly model. It is a linear model when the roll angle of ship is limited into a certain range. Conolly model can be presented by a two rank differential equation shown below [4].

$$
\left(I_{x}+\Delta I_{x}\right) \ddot{\phi}+2 N_{u} \dot{\phi}+D h \phi=-K_{w}-K_{c}
$$

Where $\phi$ is roll angle. $I_{x}$ and $\Delta I_{x}$ are inertia and added inertia relative to vertical axis of gravity center. $2 N_{u}$ is the damp coefficient of roll motion. $D$ is the tonnage of ships. $h$ is the height of transverse stability center. $K_{c}$ is the control torque produced by fin stabilizers. $K_{w}$ is the disturbance produced by ocean waves.

For fin stabilizers at zero speed, $K_{c}$ can be expressed as follows [2]:

$$
K_{c}=\left(k_{1} \omega^{2}+k_{2} \dot{\omega}\right) \cos \alpha
$$

Where $k_{1}$ and $k_{2}$ are constants. $\omega$ is angular velocity of fin stabilizers. $\alpha$ is the angle between fin stabilizers and horizontal position. Controller changes $K_{c}$ by adjusting the operating modes of fin stabilizers, and then roll motion is reduced by keeping both sides of equation 1 to zero.

\section{Optimal controller design}

Linear Quadratic Gaussian (LQG) control is the most widely used algorithm in random optimal control. Random disturbance and measurement noises can be restrained by LQG controller [5]. It's feasible to apply LQG algorithm on roll movement control of ships [6]. In order to realize LQG control, the state function of controlled system should be established, and the frequency spectrum of random wave should also be analyzed deeply.

\subsection{Establishment of state function}

By setting $x_{01}=\phi$ and $x_{02}=\dot{\phi}$, equation 1 can be modified into state functions as follows:

$$
\dot{x}_{0}(t)=A x_{0}(t)+B u_{0}(t)+C w(t)
$$

where $A=\left[\begin{array}{cc}0 & 1 \\ -D h /\left(I_{x}+\Delta I_{x}\right) & -2 N_{u} /\left(I_{x}+\Delta I_{x}\right)\end{array}\right]$, 


$$
B=\left[\begin{array}{c}
0 \\
-1 /\left(I_{x}+\Delta I_{x}\right)
\end{array}\right], C=\left[\begin{array}{c}
0 \\
-1 /\left(I_{x}+\Delta I_{x}\right)
\end{array}\right] .
$$

$u_{0}(t)$ is the input of system, and it is $K_{c}$ in equation 1. $w(t)$ is the disturbance produced by random ocean waves.

Gyroscope is the most widely used angular velocity sensor for traditional fin stabilizer control system. The roll movement of ships can be measured by gyroscope, and then the signals are transported to controller in the form of voltage. So the output of system is:

$$
y_{0}(t)=H x_{0}(t)+v(t)
$$

where $H$ is a $1 \times 2$ dimensions output matrices, and $H=\left[\begin{array}{ll}0 & 1\end{array}\right] \cdot y_{0}(t)$ is the output of angular velocity sensor. $v(t)$ is the measurement noise of angular velocity sensor. $w(t)$ is the noise of control system which is independent to $v(t)$. The variance and covariance between them are shown in equation 5 .

$$
\begin{aligned}
& E\left\{w(t) w^{T}(t)\right\}=W \\
& E\left\{v(t) v^{T}(t)\right\}=V \\
& E\left\{w(t) v^{T}(t)\right\}=0
\end{aligned}
$$

Roll movement of a ship is caused by ocean waves, so frequency spectrum of random waves should be analyzed in detail to reduce roll movement effectively. For a ship, disturbances produced by fully developed ocean waves are a series of stationary random processes. Random optimal control theory is suitable for roll controller of ships. Kalman filter is very effective to estimate state variables when the disturbances of controlled system are random. If the disturbances of system are not Gaussian white noises, Kalman filter is not optimal [7]. Long-crest random wave is approximate to Gaussian distribution. Its spectrum is a narrow-banded spectrum, and it is a kind of colored noises which is approximate to Gaussian distribution. To solve this problem, the ocean wave spectrum is fitted by modeling filter. Thus modeling filter can be seen as a part of control system. The disturbance produced by random waves is the output of modeling filter which is driven by white noises. The disturbance of extended system can be seen as white noises which are filtered by Kalman filter. The modeling filter for roll reduction can be expressed as follows according to experience [4]. The parameters of modeling filter are relative to intensity of ocean waves.

$$
G_{f}(s)=\frac{b_{1} s}{s^{2}+a_{1} s+a_{2}}
$$

Equation 6 can be modified into state function in equation 7.

$$
\left\{\begin{array}{c}
\dot{m}(t)=A_{f} m(t)+C_{f} w_{f}(t) \\
y_{f}(t)=H_{f} m(t)
\end{array}\right.
$$

where $A_{f}=\left[\begin{array}{cc}0 & 1 \\ -a_{1} & -a_{2}\end{array}\right], C_{f}=\left[\begin{array}{l}0 \\ 1\end{array}\right], H_{f}=\left[\begin{array}{ll}0 & b_{1}\end{array}\right]$.

$m(t)$ is the intermediate variable of modeling filter that has no physical meanings. $w_{f}(t)$ is zero mean white noise. $y_{f}(t)$ is colored noise outputted by modeling filter which is the disturbance $w(t)$ in equation 3. The parameters of modeling filter are determined by spectrum of ocean waves. Considering equation 3 , equation 4 and equation 7 , they can be combined into an extended state function which is shown in equation 8.

$$
\left\{\begin{array}{c}
\dot{x}(t)=\bar{A} x(t)+\bar{B} u(t)+\bar{C} w_{f}(t) \\
y(t)=\bar{H} x(t)+v(t)
\end{array}\right.
$$

where $x=\left[\begin{array}{l}x_{0}(t) \\ m(t)\end{array}\right], \bar{A}=\left[\begin{array}{cc}A & C H_{f} \\ 0 & A_{f}\end{array}\right], \bar{B}=\left[\begin{array}{l}B \\ 0\end{array}\right]$, $\bar{C}=\left[\begin{array}{c}0 \\ C_{f}\end{array}\right], \bar{H}=\left[\begin{array}{ll}H & 0\end{array}\right]$.

\subsection{Optimal control of fin stabilizer}

Diagram of optimal control system for fin stabilizer at zero speed based on equation 8 is shown in Fig.3.

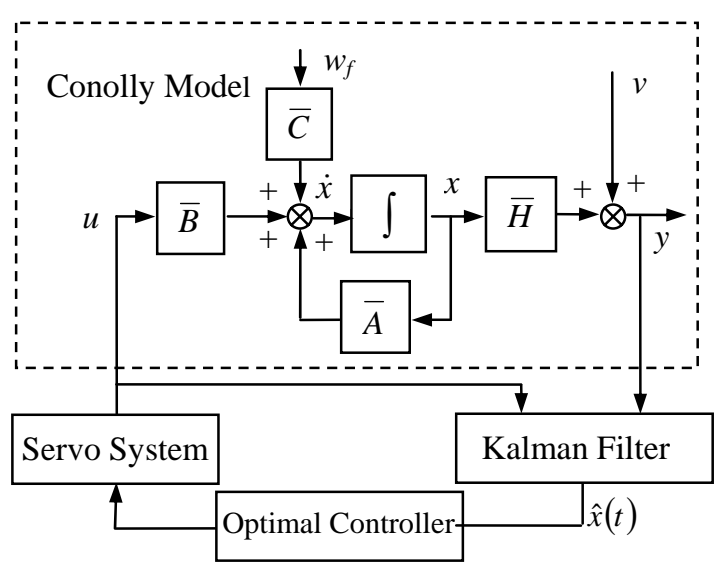

Fig.3: Random optimal control of roll motion.

The purpose of optimal control is to minimize the performance index by introducing optimal algorithm. 
For optimal control of fin stabilizer at zero speed, performance index can be expressed as follows:

$$
J=E\left\{\frac{1}{2} \int_{0}^{\infty}\left[\lambda x^{T}(t) Q(t) x(t)+u^{T}(t) R(t) u(t)\right] d t\right\}
$$

where $Q$ is the weight matrix of state variable. It is a symmetrical semi-definite matrix. $Q$ reflects dynamic error of control system. $R$ is the weight matrix of input. It is a symmetrical definite matrix and reflects the convergence rate of state variable and energy consumption of system [8]. The physical meaning of performance index is to keep the roll angle of ship within a certain range with least energy. That is to say, dynamic performance and energy consumption are optimized together. When the dimension of system is very high, it is too complicated to determine the parameters of weight matrices without optimal searching algorithm. There might be great difference between the magnitude of state variable $x(t)$ and input $u(t)$. Optimal searching algorithm can only guarantee that the performance index reaches minimum value. As a result, the difference between the first part and the second part of index might be very great. $x(t)$ and $u(t)$ cannot be optimized effectively at the same time. For example, if $x(t)$ is too small, the first part of performance index is much smaller than the second part. The optimization of $x(t)$ is weaken. Thus a weighting factor $\lambda$ is introduced to balance the value of the two parts.

Optimal control system can be departed into two parts based on separation theorem. One part is definite optimal controller, the other part is optimal filter. Optimal control rules can be expressed as:

$$
u(t)=-K_{c} \hat{x}(t)
$$

Feedback gain matrix is derived based on definite optimal control rules, i.e.

$$
K_{c}=R^{-1} \bar{B}^{T} P_{c}
$$

Where $P_{c}$ can be calculated from the following algebraic Riccati equation.

$$
\bar{A}^{T} P_{c}+P_{c} \bar{A}-P_{c} \bar{B} R^{-1} \bar{B}^{T} P_{c}+Q=0
$$

For a linear control system with random disturbance, its optimal control rules are the same with corresponding definite system without disturbance. The difference between them is that the minimum variance tracking $\hat{x}(t)$ replaces the real state variable $x(t) . \hat{x}(t)$ is determined by Kalman filter function as follows:

$$
\hat{\dot{x}}(t)=\bar{A} \hat{x}(t)+\bar{B} u(t)+K_{f}[y(t)-\bar{H} \hat{x}(t)]
$$

The gain of Kalman filter $K_{f}$ is calculated as follows:

$$
K_{f}=P_{f} \bar{H}^{T} V^{-1}
$$

where $P_{f}$ can be calculated from the following algebraic Riccati equation.

$\bar{A}^{T} P_{f}+P_{f} \bar{A}-P_{f} \bar{B} V^{-1} \bar{B}^{T} P_{f}+\bar{C} W \bar{C}^{T}=0$

Optimal control rules are obtained by establishing the feedback loop with optimal controller and Kalman filter.

\section{Optimization of weight matrices}

\subsection{Optimizing weight matrices with genetic algorithm}

If the weight matrix is determined improperly, control system cannot be optimized as expected. The parameters of weight matrices are usually determined according to experiences of designers [9]. Parameters must be improved many times to obtain expected performance. The application of LQG control is restrained for this disadvantage. It is hard to determine the weight matrix according to experience, because too many parameters are involved for extended system shown in equation 8. So genetic algorithm is introduced to optimize the parameters of weight matrices.

Genetic algorithm has the same principle with natural selection and heredity in biology. Genetic algorithm needs little information and simple calculation to obtain global optimal solutions [10]. As is shown in Fig.4, there are three steps to optimize the weight matrix of performance index. It is named as selection, cross and variation. Selection is to choose the individuals with better adaptability. Cross is to exchange part of two parent individuals to reform a new individual. Cross is to change a part of an individual in certain probability to avoid the algorithm obtaining local optimal solution.

There are two important problems to be concerned when weight matrices are optimized with genetic algorithm. One is the coding of matrices, the other is the definition of fitness function.

Individuals are expressed in binary symbol strings in basic genetic algorithm. But the effectiveness of binary coding is not acceptable when optimizing mutidimension and high accuracy systems. Thus floating point numbers are used to coding individuals in roll movement control system, because the parameters of weight matrices are real numbers. It is more efficient 
and accurate than binary coding, because number system conversion is omitted [10].

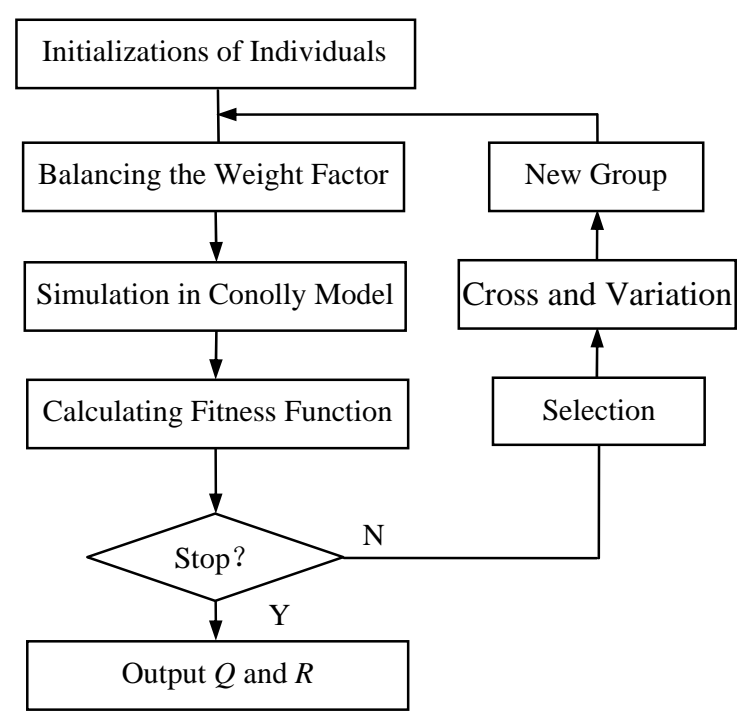

Fig.4: Flow chart of acquiring weight matrices with genetic algorithm.

External information is seldom used when searching a solution with genetic algorithm. The algorithm is evaluated by fitness function. The convergence rate of algorithm is greatly influenced by fitness function. The aim of genetic algorithm is to search individuals with maximum fitness function value which is positive. For optimal control of fin stabilizer at zero speed, the performance index $J$ is a positive function. But the purpose of optimization is to minimize performance index. So the reciprocal of index can be used as fitness function. That is to say, the fitness function $f$ is stated as : $f=1 / J$.

\subsection{Balancing performance index with weight factor}

Simulation on a certain ship is taken as an example when the weight matrices are determined according to experience. The parameters of ship is [11]: $\mathrm{D}=1.4572$ $\times 10^{6} \mathrm{~kg}, \mathrm{~h}=1.15 \mathrm{~m}, I_{x}+\Delta I_{x}=3.4383 \times 10^{6} \mathrm{~kg} \cdot \mathrm{m}^{2}$, $N_{u}=3.18 \times 10^{5}$. Simulation results indicate that the input of the system is about $10^{5} \mathrm{~N} \cdot \mathrm{m}$, but the angle of ship usually vary between $-5^{\circ}$ and $+5^{\circ}$. So the relation between the norm of state variable and input can be expressed as follows:

$$
\|x(t)\|_{\infty}<<\|u(t)\|_{\infty}
$$

The aim of genetic algorithm is to minimize the performance index. If the parameters of weight matrices are optimized by basic genetic algorithm, the second part of performance index will be much greater than the first part. Thus only the energy consumption of system is optimized, and the optimization of roll movement is almost neglected. So the value of two weight matrices must be balanced. $Q$ is a symmetrical matrix, and can be expressed as: $Q=\operatorname{diag}\left[q_{1}, q_{2}, q_{3}, q_{4}\right]$. The balanced matrix is shown as follows:

$$
Q^{\prime}=\lambda Q=\lambda \cdot \operatorname{diag}\left[q_{1}, q_{2}, q_{3}, q_{4}\right]
$$

where $\lambda$ is calculated as follows to keep the two parts of performance index in the same order.

$$
\lambda=\frac{\|u(t)\|_{\infty}}{\|x(t)\|_{\infty}}
$$

The other weight matrix $R$ is actually a real number, and can be stated as: $R=r$. Thus the optimization of weight matrices is to optimize the following five parameters: $q_{i} \quad(i=1,2,3,4)$ and $r$. The parameters are optimized under five and six level waves respectively. The scale of group is 30. Cross probability is 0.5 . Variation probability is 0.05 . The weight matrix is obtained after 25 generations of calculation,i.e $Q_{5}=10^{10} \times \operatorname{diag}[0.21,0.07,3.10,2.63], R_{5}$ $=0.51, Q_{6}=10^{10} \times \operatorname{diag}[0.35,0.02,4.12,1.71], R_{6}=0.57$.

\subsection{Adjusting weight matrices with intensity of ocean waves}

The intensity of waves varies with time, and the parameters of Conolly model mentioned above will change with the load of ships. So the two weight matrices must be adjusted with external environments. Thus control rules are suitable to in any conditions. The weight matrices should be adjusted according to some adaptive rules, i.e.

- Roll angle of ship, rotating angle and angular velocity of fin stabilizer must be limited in certain ranges; "Bullet” style, bullet style, bullet style

- When roll angle of ship is too small, it means that too much energy is consumed to reduce roll movement. The output of controller should be reduced to save energy and avoid mechanism abrasion;

- The output of controller should be reduced when rotating angle and angular velocity of fin stabilizer reach their boundaries frequently;

- The output of controller should be enhanced when the roll angle of ship is too large.

Statements above are just qualitative analysis of adaptive rules. The rules should be quantitatively expressed in engineering. When the intensity of ocean 
waves is low, the average roll angle of ship $\bar{\phi}$ and the maximum rotating angular velocity of fin stabilizer $\omega_{\max }$ are also small. But energy consumption and mechanism abrasion are enhanced if $\bar{\phi}$ is too small. When the intensity of ocean waves is high, $\bar{\phi}$ and $\omega_{\max }$ increase. If fin stabilizer reaches its boundary frequently at very high speed, the mechanism system is likely to be damaged. So the output of controller must be adjusted properly on line based on the intensity of ocean waves. As is shown in equation 2, control torque $K_{c}$ reflects the intensity of ocean waves. That is to say, intensity of ocean waves is the function of $\omega_{\max }$. The weight matrices can be adjusted based on $\omega_{\max }$. When the intensity of ocean waves is invariable, the weight matrices is adjusted based on required $\bar{\phi}$.

Adaptive rules analyzed above can be transformed into fuzzy control rules. Fuzzy control rule table is obtained by optimizing the weight matrices in different intensity of waves with genetic algorithm. Classification of $\bar{\phi}$ and $\omega_{\max }$ in Table 1 is based on the data measured from the sailing ship.

\begin{tabular}{|c|c|c|c|}
\hline \multirow{2}{*}{$\omega_{\max }$} & \multicolumn{3}{|c|}{$\bar{\phi}$} \\
\cline { 2 - 4 } & $\bar{\phi}_{1}$ & $\bar{\phi}_{2}$ & $\bar{\phi}_{3}$ \\
\hline$\omega_{\max 1}$ & $\left(Q_{11}, R_{11}\right)$ & $\left(Q_{12}, R_{12}\right)$ & $\left(Q_{13}, R_{13}\right)$ \\
\hline$\omega_{\max 2}$ & $\left(Q_{21}, R_{21}\right)$ & $\left(Q_{22}, R_{22}\right)$ & $\left(Q_{23}, R_{23}\right)$ \\
\hline$\omega_{\max 3}$ & $\left(Q_{31}, R_{31}\right)$ & $\left(Q_{32}, R_{32}\right)$ & $\left(Q_{33}, R_{33}\right)$ \\
\hline
\end{tabular}

Table 1: Fuzzy control rule table.

When the roll angle of ship is smaller than $\bar{\phi}_{1}$, weight matrices are selected from the first column. If roll angle of ship is too small at this moment, the gain between controller and servo system is reduced. Thus the second rule of adaptive rules is realized. When the roll angle of ship is between $\bar{\phi}_{1}$ and $\bar{\phi}_{2}$, weight matrices are selected from the second column. When the roll angle of ship is larger than $\bar{\phi}_{2}$, the gain between controller and servo system is enhanced. Thus the forth rule of adaptive rules is realized. The weight matrices are selected in the same way in other conditions. What's more, the third rule is also realized by adjusting the gain.

The optimization of roll movement and energy are incompatible each other. They cannot be optimal at the same time. But the best matching point of them can be found if the fuzzy control rule in Table 1 is established appropriately.

\section{Simulation}

If the roll angle of ship is smaller than $\bar{\phi}_{1}$, it is assumed that the intensity of ocean waves is five level when $\omega_{\max }$ is between $\omega_{\max 1}$ and $\omega_{\max 2}$, and the intensity of ocean waves is six level when $\omega_{\max }$ is between $\omega_{\max 2}$ and $\omega_{\max 3}$. Thus $\left(Q_{5}, R_{5}\right)$ and $\left(Q_{6}, R_{6}\right)$ are $\left(Q_{21}, R_{21}\right)$ and $\left(Q_{31}, R_{31}\right)$ in Table 1 respectively. The ending time of performance index in equation 9 is infinite. The value of index will be infinite if the system is simulated as equation 9. So only the index value in finite time is calculated in engineering. Equation 9 can be discretized as follows:

$$
J=E\left\{\frac{1}{2} \sum_{k=0}^{N-1}\left[x^{T}(k) Q x(k)+u^{T}(k) R u(k)\right]\right\}
$$

Assuming $t_{f}=1200 \mathrm{~s}$ and the sampling period is $0.5 \mathrm{~s}$, the ship mentioned in 4.2 section is simulated in Matlab by selecting $\left(Q_{5}, R_{5}\right)$ and $\left(Q_{6}, R_{6}\right)$ as weight matrices. The roll angle of ship is shown in Fig.5. It can be seen that no matter what matrices are selected, the angle of ship varies between $-5^{\circ}$ and $+5^{\circ}$. The magnitude of roll angle meets the requirement.

Control torque produced by fin stabilizer is shown in Fig.6. The value of control torque corresponding to $Q_{5}$ and $R_{5}$ is lower than that corresponding to $Q_{6}$ and $R_{6}$. Lower control torque means less energy consumption. So driving energy can be optimized by selecting proper weight matrices. The value of performance index calculated from equation 19 is: $J_{5}=1.8478 \times 10^{14}, J_{6}=1.9776 \times 10^{14} . J_{5}$ is smaller than $J_{6}$. It can be concluded that $Q_{5}$ and $R_{5}$ are proper matrices for five level waves.

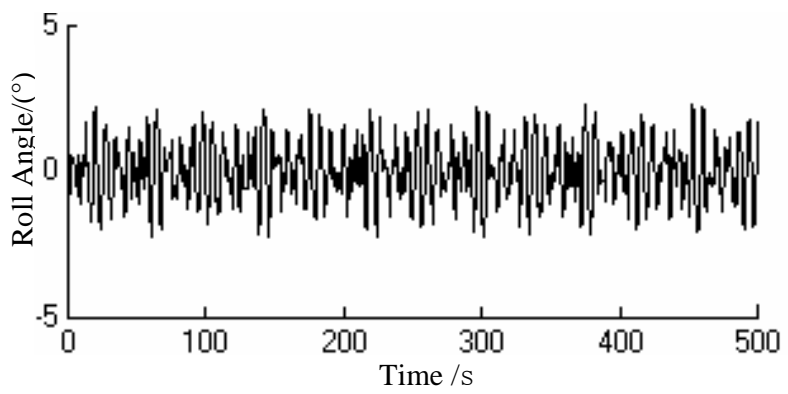

(a) $Q_{5}$ and $R_{5}$ are selected as weight matrices. 


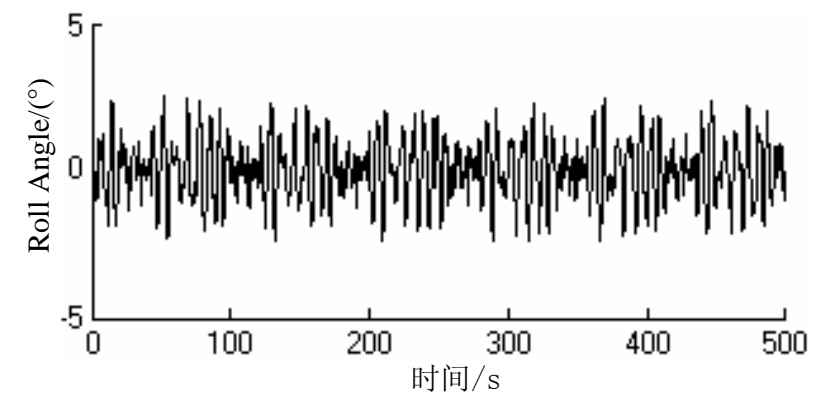

(b) $Q_{6}$ and $R_{6}$ are selected as weight matrices.

Fig.5: Roll angle of ship in five level waves.

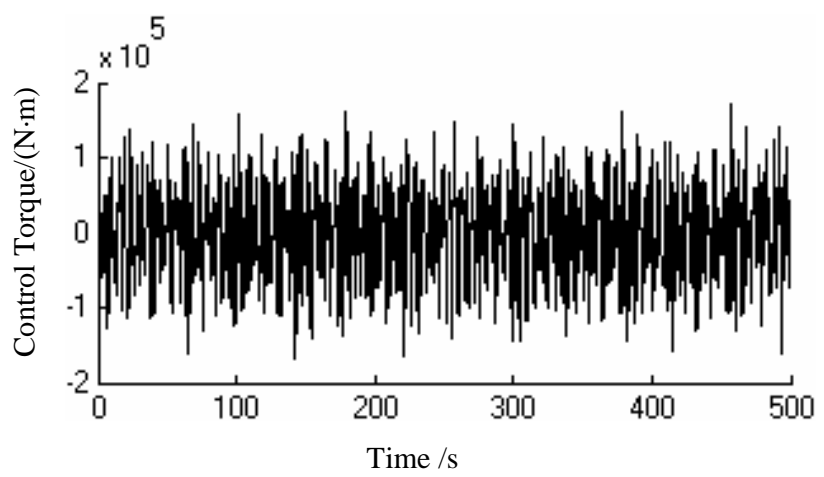

(a) $Q_{5}$ and $R_{5}$ are selected as weight matrices.

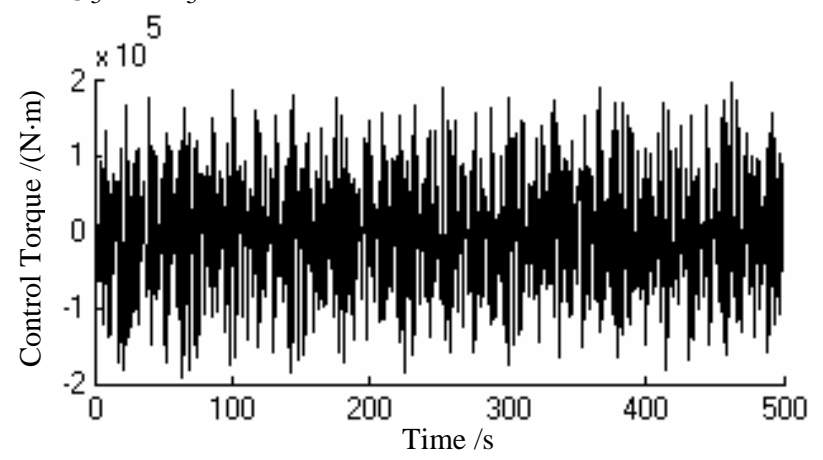

(b) $Q_{6}$ and $R_{6}$ are selected as weight matrices.

Fig.6: Control torque of fin stabilizer in five level waves.

If $\left(Q_{5}, R_{5}\right)$ and $\left(Q_{6}, R_{6}\right)$ are selected as weight matrices, the value of performance index is: $J_{5}=3.0885 \times 10^{14}, J_{6}=2.8871 \times 10^{14}$. $J_{6}$ is smaller than $J_{5} \cdot Q_{6}$ and $R_{6}$ are proper matrices for six level waves. $Q_{5}$ and $R_{5}$ are not proper matrices.

The expression in equation 9 is the performance index on roll movement and energy consumption. Both of them can be optimized if the weight matrices are chosen appropriately.

\section{Conclusions}

Frequency spectrum of random wave is analyzed in order to enhance the efficiency of fin stabilizers at zero speed when the intensity of ocean wave is changing. Optimal control rules based on quadratic performance index are obtained by analyzing the feature of ocean waves. Performance index weight matrices are optimized with genetic algorithm to find the best matching point between energy consumption and effect of roll reduction. Basic genetic algorithm is improved by analyzing the characteristic of the system. It can be concluded that energy consumption can be optimized if the weight matrices of index is selected appropriately according to the intensity of ocean waves.

\section{Acknowledgement}

This work is supported by National Nature Science Foundation of China (Grant No.50575048) and Postdoctor Foundation of Heilongjiang Province Government (Grant No. LBH-Z05052).

\section{References}

[1] R.P. Dallinga, Roll stabilization of motor yacht: Use of fin stabilizers in anchored conditions. Project 99, Amsterdam, 1999.

[2] R.P. Dallinga, Roll stabilization of motor yacht: Hydrodynamic aspects of the comparison of antiroll tanks and fins. Project 2002, Amsterdam, 2002.

[3] J.Ooms, The use of roll stabilizer fins at zero speed. Project 2002, Amsterdam, 2002.

[4] H.Z. Jin, X.L. Yao, Control Method Applied to Ships, Harbin: Harbin Engineering University Press, pp.140-143, 2001.

[5] W.W. Chen, Y. Wang and Q.R. Wang, Adaptive LQG control for the electric power steering system of an mobile, Journal of Mechanism Engineering, 41(12):167-172, 2005.

[6] H.L. Chen, X.R. Zhao and K. Ye, Using LQG control method for pitch stabilization of ships, Journal of Harbin Engineering University, 25(4):407-411, 2004.

[7] S.S. Hu, Z.Q. Wang and W.L. Hu, Optimal Control and System, Beijing: Science Press, 2005.

[8] M.X. Fang, Q. Feng and X. Ma, Random Optimal Control for MAGLEV Train, Chinese Quarterly of Mechanics. 24(2):174-178, 2003.

[9] H.T. Lin, M. Wang and L. Chen, PID Parameter Adjustment Based on Genetic Algorithm, Journal of Shenyang Institute of Technology, 23(4):27-30, 2004. 
[10] S.H. Wang, D.Q. Shu, Intelligent Control System and Its Application, Beijing: Mechanic Press, 2005.

[11] H.P. Zhang, X.L. Yao and H.Z. Jin, Robust Fin Stabilizer With Lifting Force Feedback, Control Theory and Applications, 22(9):15-18, 2002. 